Check for updates

Cite this: Phys. Chem. Chem. Phys., 2020, 22, 17008

OI: $10.1039 / \mathrm{d} 0 \mathrm{cp} 90155 \mathrm{e}$

rsc.li/pccp

\section{Correction: High pressure single-molecule FRET studies of the lysine riboswitch: cationic and osmolytic effects on pressure induced denaturation}

\author{
Hsuan-Lei Sung ${ }^{\mathrm{ab}}$ and David J. Nesbitt*abc \\ Correction for 'High pressure single-molecule FRET studies of the lysine riboswitch: cationic and \\ osmolytic effects on pressure induced denaturation' by Hsuan-Lei Sung et al., Phys. Chem. Chem. Phys., \\ 2020, DOI: 10.1039/d0cp01921f.
}

Correction \#1: The apportioning of support in the acknowledgements was incorrectly captured in the original document. The corrected acknowledgements should read:

\title{
Acknowledgements
}

Primary support for this work has been provided by the National Science Foundation under grant CHE-1665271 from the Chemical, Structure, Dynamics and Mechanisms-A Program, with additional support for development of the confocal apparatus from PHY-1734006 (Physics Frontier Center Program). We would also like to acknowledge early seed contributions by the W. M. Keck Foundation Initiative in RNA Sciences at the University of Colorado, Boulder, and well as transitional support from the Air Force Office of Scientific Research (FA9550-15-1-0090).

Correction \#2: Furthermore, the writing and submission of this paper occurred in the first weeks of the COVID-19 shutdown for JILA and the University of Colorado in Boulder, requiring the authors to transfer multiple Word and EndNote files from a lab computer to a home computer. In the process an EndNote library was corrupted, which resulted in several errors in the referencing process not caught until the article appeared online. Below, we summarize corrections in the references, apologizing for any challenges this creates for the reader. A fully corrected pdf is available on request (and on the Nesbitt group website, https://jila.colorado.edu/nesbitt/).

\begin{tabular}{lll}
\hline Location & Original reference given in published paper & Replacement reference from list of new references below \\
\hline Page 2 & Ref. 37 & Ref. 1 \\
Page 2 & Ref. 38 & Ref. 2 \\
Page 2 & Ref. 40 & Ref. 3 \\
Page 2 & Ref. 41 & Ref. 4 \\
Pages $4,7,8(\times 2)$ and 11 Ref. 10 & Ref. 5 \\
Pages 6 and 9 & Ref. 53 & Ref. 6 \\
Page 6 & Ref. 54 & Ref. 7 \\
Page 7 & Ref. 58 & Ref. 8 \\
Page 7 & Ref. 59 & Ref. 9 \\
Pages 7 and 9 & Ref. 60 & Ref. 10
\end{tabular}

${ }^{a}$ JILA, National Institute of Standards and Technology and University of Colorado, Boulder, CO 80309, USA. E-mail: djn@jila.colorado.edu

${ }^{b}$ Department of Chemistry and Biochemistry, University of Colorado, Boulder, CO 80309, USA

${ }^{c}$ Department of Physics, University of Colorado, Boulder, CO 80309, USA 
Pages 7 and 9

Pages 7 and 9

Page $9(\times 2)$

Page 10

Page 11

Page $11(\times 2)$

Page $11(\times 3)$

Page $11(\times 2)$
Ref. 61

Ref. 65

Ref. 71

Ref. 73

Ref. 11

Ref. 75

Ref. 76

Ref. 77
Ref. 11

Ref. 12

Ref. 13

Ref. 14

Ref. 15

Ref. 16

Ref. 17

Ref. 18

On page 1, ref. 11 should be deleted.

On page 6, ref. 55 should be replaced with ref. 11 from the published article.

The Royal Society of Chemistry apologises for these errors and any consequent inconvenience to authors and readers.

\section{References}

1 T. O. Street, D. W. Bolen and G. D. Rose, A Molecular Mechanism for Osmolyte-Induced Protein Stability, Proc. Natl. Acad. Sci. U. S. A., 2006, 103, 13997-14002.

2 D. R. Canchi and A. E. García, Cosolvent Effects on Protein Stability, Annu. Rev. Phys. Chem., 2013, 64, $273-293$.

3 D. Lambert, D. Leipply and D. E. Draper, The Osmolyte TMAO Stabilizes Native RNA Tertiary Structures in the Absence of Mg ${ }^{2+}$ : Evidence for a Large Barrier to Folding from Phosphate Dehydration, J. Mol. Biol., 2010, 404, 138-157.

4 P. H. Yancey and J. F. Siebenaller, Trimethylamine Oxide Stabilizes Teleost and Mammalian Lactate Dehydrogenases Against Inactivation by Hydrostatic Pressure and Trypsinolysis, J. Exp. Biol., 1999, 202, 3597-3603.

5 R. d. C. Barbosa and M. C. Barbosa, Hydration Shell of the TS-Kappa Protein: Higher Density than Bulk Water, Phys. A, 2015, 439, 48-58.

6 D. E. Draper, D. Grilley and A. M. Soto, Ions and RNA Folding, Annu. Rev. Biophys. Biomol. Struct., 2005, 34, $221-243$.

7 D. E. Draper, RNA Folding: Thermodynamic and Molecular Descriptions of the Roles of Ions, Biophys. J., $2008,95,5489-5495$.

8 N. Tsukida, H. Muranaka, M. Ide, Y. Maeda and H. Kitano, Effect of Neutralization of Poly(acrylic acid) on the Structure of Water Examined by Raman Spectroscopy, J. Phys. Chem. B, 1997, 101, 6676-6679.

9 E. D. Holmstrom, J. L. Fiore and D. J. Nesbitt, Thermodynamic Origins of Monovalent Facilitated RNA Folding, Biochemistry, 2012, 51, 3732-3743.

10 J. C. Schlatterer, L. W. Kwok, J. S. Lamb, H. Y. Park, K. Andresen, M. Brenowitz and L. Pollack, Hinge Stiffness is a Barrier to RNA Folding, J. Mol. Biol., 2008, 379, 859-870.

11 T. J. Wilson and D. M. J. Lilley, Metal Ion Binding and the Folding of the Hairpin Ribozyme, RNA, 2002, 8, 587-600.

12 R. Shiman and D. E. Draper, Stabilization of RNA Tertiary Structure by Monovalent Cations, J. Mol. Biol., $2000,302,79-91$.

13 S. L. Heilman-Miller, D. Thirumalai and S. A. Woodson, Role of Counterion Condensation in Folding of the Tetrahymena Ribozyme. I. Equilibrium Stabilization by Cations, J. Mol. Biol., 2001, 306, 1157-1166.

14 J. J. Childress and B. A. Seibel, Life at Stable Low Oxygen Levels: Adaptations of Animals to Oceanic Oxygen Minimum Layers, J. Exp. Biol., 1998, 201, 1223-1232.

15 R. J. Ellis, Macromolecular Crowding: Obvious but Underappreciated, Trends Biochem. Sci., 2001, $26,597-604$.

16 J. Ma, I. M. Pazos and F. Gai, Microscopic Insights into the Protein-Stabilizing Effect of Trimethylamine N-Oxide (TMAO), Proc. Natl. Acad. Sci. U. S. A., 2014, 111, 8476-8481.

17 D. L. Pincus, C. Hyeon and D. Thirumalai, Effects of Trimethylamine $N$-Oxide (TMAO) and Crowding Agents on the Stability of RNA Hairpins, J. Am. Chem. Soc., 2008, 130, 7364-7372.

18 Q. Zou, B. J. Bennion, V. Daggett and K. P. Murphy, The Molecular Mechanism of Stabilization of Proteins by TMAO and its Ability to Counteract the Effects of Urea, J. Am. Chem. Soc., 2002, 124, 1192-1202. 